\title{
Predictors of Outcome in Diabetic Foot: A Narrative Review
}

\author{
Bagavathi Vinuthi $\mathrm{M}^{1}$, Dinesh Rajasekar $\mathrm{AV}^{2}$, Vinoth Kumar $\mathrm{S}^{3}$, Ganesh Babu $\mathrm{CP}^{4}$
}

\begin{abstract}
Background: Diabetes is a life-changing disease associated with the severe morbidity and also mortality. Among the diabetic patients, the prevalence of foot ulcers will usually be as high as $20 \%$. These ulcers usually become infected, cause very high morbidity, and are the usual first step for lower extremity amputation. The holistic care of diabetic foot ulcers requires a multidisciplinary approach. Apart from blood sugar control, treatment of ulcer involves wound debridement, appropriate dressings, vascular maintenance, and control of infections. The most important factor in preventing amputation is a repeated education of the patient in foot care and to really look into factors associated with amputation. This review article is an attempt to identify the statistically significant risk factors leading to lower extremity amputation.

Materials and methods: The research literature was searched for various studies reporting the risk factors for lower extremity amputation in patients with DFI. A systematic search of literature was conducted first in MEDLINE/PubMed and then in Cochrane Central Register of Controlled Trials till July 2019. This search retrieved 11 most relevant articles. The risk factors that led to LEA were studied and data were analyzed.

Results: Based on the comparison, we have concluded that male preponderance, duration of diabetes, and vasculopathy were found to be statistically significant in majority of the studies.

Keywords: Diabetic foot infection, Diabetic foot ulcer, Lower extremity amputation, Peripheral arterial disease.

Annals of SBV (2019): 10.5005/jp-journals-10085-8112
\end{abstract}

\section{INTRODUCTION}

The prevalence of diabetes mellitus (DM) is rising dramatically at present. ${ }^{1}$ The World Health Organization has proclaimed an estimated number of patients with diabetes was nearly 425 million in 2017 and consequently, increasing the number of diabetes-related complications. ${ }^{2}$ These complications have led to a decrease in quality of life and increase in financial costs, morbidity, and even mortality. The development of foot ulcers, one of the dominant complications of diabetes mellitus, is $15-25 \%{ }^{3}$ Diabetic foot ulceration is a major cause of nontraumatic lower extremity amputations. ${ }^{4}$ Exceeding number of diabetic foot ulcers undergoes infection, which paves way for higher amputation rates in the diabetes population. ${ }^{5}$ Hence, diabetic foot infections (DFIs) account for greater hospitalization and limb loss and thus have a compelling effect on quality of life. ${ }^{6}$ Diagnosis and management of infection in the foot are crucial because of expanded prevalence and the concern toward health in diabetic patients. Consequently, familiarity toward factors that cause exposure is essential to establish diagnosis along with management and protocols for treating and prevention of the lower extremity amputation for patients with DFls. ${ }^{7}$ Multiple risk factors for lower extremity amputation in patients with diabetes have been explored, like peripheral arterial disease (PAD), peripheral neuropathy and osteomyelitis, and the corelated morbidities. Despite certain factors remain unclear those which are vital and to a limit for which they escalade the risk of amputation. ${ }^{8}$ Inspite of the published research on multiple risk factors for the lower extremity amputations associated to DFI, none has been analyzed methodically in a meta-analysis. So this study reassesses multiple causes, which advance to lower extremity amputation in patients with diabetic foot.

\section{Materials and Methods}

The research literature was searched for studies reporting risk factors for lower extremity amputation in patients with DFI. The literature was searched in a systematic order conducted first

\begin{abstract}
${ }^{1-4}$ Department of General Surgery, Mahatma Gandhi Medical College and Research Institute, Sri Balaji Vidyapeeth (Deemed to be) University, Puducherry, India

Corresponding Author: S Vinoth Kumar, Department of General Surgery, Mahatma Gandhi Medical College and Research Institute, Sri Balaji Vidyapeeth (Deemed to be) University, Puducherry, India, Phone: +918110011955, e-mail: vino8683@gmail.com
\end{abstract}

How to cite this article: Bagavathi Vinuthi M, Dinesh Rajasekar AV, Vinoth Kumar S, Ganesh Babu CP. Predictors of Outcome in Diabetic Foot: A Narrative Review. Ann SBV 2019;8(2):42-44.

Source of support: Sri Balaji Vidyapeeth University

Conflict of interest: None

in the MEDLINE/PubMed and later in Cochrane Central Register of Controlled Trials till December 2019. Strategies of the search included related keywords for the three concepts: "diabetic foot," "infection," and "lower extremity amputation." This search retrieved 20 most relevant articles. They were taken into account in the review if it satisfied the forthcoming selection criteria: (i) theme: studies that estimated the relation between the variables (clinical data or diagnostic tests results) and DFU; (ii) type of study: cross-sectional studies; (iii) results: studies must end if no statistically relevant relationship between independent variables and DFU.

\section{Study Selection}

Appropriate articles for the narrative review were the studies over the risk factors for amputation in patients with DFI. Principle result variables comprised of minor and major amputations. Cross-sectional studies were only included. We also screened the reference lists of selected studies manually for the applicable articles. Articles were excluded if include studies without complete text, studies without assessment of risk factors for amputations, or with evaluations of diabetic foot ulcers. Every risk factors that were mentioned in the included study were analyzed in present study.

(O) The Author(s). 20190pen Access This article is distributed under the terms of the Creative Commons Attribution 4.0 International License (https://creativecommons. org/licenses/by-nc/4.0/), which permits unrestricted use, distribution, and non-commercial reproduction in any medium, provided you give appropriate credit to the original author(s) and the source, provide a link to the Creative Commons license, and indicate if changes were made. The Creative Commons Public Domain Dedication waiver (http://creativecommons.org/publicdomain/zero/1.0/) applies to the data made available in this article, unless otherwise stated. 
Total no of studies reclaimed

by electronic search $(n=151)$

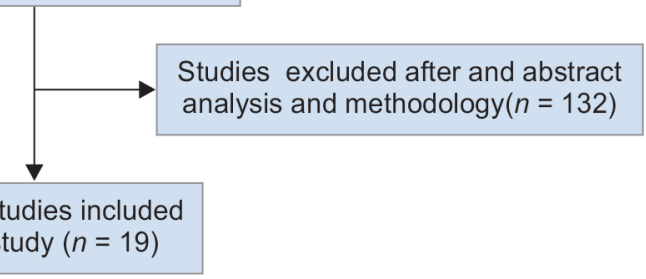

The various risk factors which were studied included age, duration of diabetes, $\mathrm{ABPI}$, neuropathy, nephropathy, vasculopathy, hyperlipidemia, $\mathrm{HbA1c}$, smoking, and bone involvement.

In our study, among 100 diabetic foot ulcer patients, 40 were managed conservatively and 60 underwent amputation (both minor and major). The various risk factors which led to lower extremity amputation were studied.

\section{Discussion}

\section{Age Distribution}

Association of age with diabetic foot ulcer has been evaluated ( $n=11)$, despite results are somewhat conflicting. Armstrong et al., ${ }^{9}$ in an RCT, established that younger patients being at higher risk, while four other studies ${ }^{3,10-12}$ with consecutive patient selection achieved the opposite (older patients presented with higher risk). Two other studies 3,12 found a relation between age and active or recently healed DFU, with a lower age representing higher risk. In opposition, two studies ${ }^{10,11}$ concluded that for an active DFU history, a higher age represented a higher risk. Of the four studies $3,11,12$ that reported an association between age and DFU history, one concluded with greater than 75 years had greater risk. ${ }^{10}$

In our study, the mean age was in the fifth decade.

\section{Diabetes Duration}

All the studies reported a relation between diabetes of duration more than 10 years and the risk of LEA.

In our study also, among the amputated group, 70\% had diabetes more than 5 years and $p$ value was found to be statistically significant.

\section{Ankle-brachial Index}

In five studies, ankle-brachial pressure index less than 0.3 was associated with lower extremity amputation..$^{2,6,7,9,11,13}$ Among three studies, ankle-brachial pressure index of 0 was associated with lower extremity amputation. ${ }^{4,5,8}$ In our study, however, in the amputated group, $44 \%$ had ABPI more than 0.8 .

\section{Neuropathy}

This risk factor was found to be statistically significant in 4 out of 11 studies and was significantly associated with lower extremity amputation. ${ }^{3,4,6,7}$ Armstrong et al. ${ }^{9}$ declared by reporting the presence of single or multiple symptoms (numbness, burning, or tingling) had a sensitivity of an average of $100 \%$ to detect active or recently healed DFU. In our study, in the amputated group, $48 \%$ had neuropathy.

\section{Vasculopathy}

All reports proved a statistically important relation with lower extremity amputation. Peripheral pulses were examined clinically and then using a hand-held doppler. In our study, among the amputated group, $60 \%$ did not have peripheral pulses and $p$ value was found to be statistically significant.

\section{Nephropathy}

Half of the studies $s^{4,6,7,10,11,13}$ established a correlation of nephropathy with diabetic foot amputation. In our study, there was no statistical significance between nephropathy and lower limb amputation.

\section{Hyperlipidemia}

Only one study ${ }^{13}$ evaluated its association with LEA as the association was not statistically important. In our study also, there was no statistical significance between hyperlipidemia and lower extremity amputation.

\section{HbA1c}

Mostly studies assessing its association with DFU development showed that higher values marked a higher risk..$^{3-6,8,9,11,13}$ In our study, among the amputated group, $60 \%$ had HbA1c of more than 10.5 and it was not statistically significant.

\section{Smoking}

We chose eight studies assessing its relation with DFU; still, only two studies proved statistically significant results showing a higher prevalence of amputation in current smokers. ${ }^{8,9}$ In our study among the amputated group, $64 \%$ were smokers and it was not statistically significant.

Mehmood et al. of Pakistan in his paper about clinical profile and management results of diabetic foot ulcer in a tertiary care hospital determined that out of 120 patients, majority of patients who underwent lower limb amputation ended up to be type II diabetic patients $(95.7 \%)$, males $(66 \%)$ with a duration of diabetes of more than 10 years $(p<0.05)$. The mean age was $54.29 \pm 7.71$ years. ${ }^{12}$

Al-Maskari et al. studied the prevalence of factors which accounts for diabetic foot complications. Out of the 513 diabetic patients (a mean age of 53 years), 12\% had peripheral vascular disease. Significant risk factors were male gender, increased duration of diabetes, and presence of hypertension. ${ }^{14}$

Diabetic foot ulcers revascularize when possible and improve the flow of blood and aid wound healing. Amputation is also prevented by proper education of the diabetic patients, foot care, and appropriate footwear. This was proved by Casselini et al. in his paper on "Approach to the diabetic foot."15

Van Damme et al. studied proved that PVD is a major factor for amputation. PVD is not only involves the distal pulses but also fully involves femoral, popliteal, and tibial pulses. It can be treated by open or endovascular surgical procedures. Also Charcot's neuroarthropathy is specific for complication of neuropathy, which leads to fragmentation and destruction of the bones and joints. $^{16}$

The article of infection and diabetic foot by Alwakeel et al. found that the end result of preexisting foot wound whose chronic nature is aggravated by the factor, such as, neuropathy and PVD, which is factor of bad outcome, regarding the risk for leg amputation. ${ }^{17}$

A case-control study over risk factors for toe amputation in a diabetic population by Deshpande et al. shows that obvious risk factors for toe amputation were male sex, osteomyelitis, digital deformity, diabetic nephropathy, diabetic neuropathy, and ischemia. $^{18}$

Formosa et al. had elaborated that prevalence of infection was $6-11 \%$ imputation and $3 \%$ in study group of 1,319. Neuropathy was significant factor leading to imputation (15\%). ${ }^{19}$ 
Dalla Paola et al. discovered the separate effects of PVD, sensory neuropathy, and foot ulcer in the diabetic patients with lower extremity amputation. His study revealed that peripheral sensory neuropathy, PVD foot ulcers, and former amputation were the independent risk factors for LEA. ${ }^{20}$

\section{Conclusion}

Based on the results, we have concluded that the maximum incidence of diabetic foot ulcers in the fifth decade of life and men are most commonly affected. Age of the patients (most common in 5th decade), duration of diabetes more than 5 years, and vasculopathy are statistically significant risk factors for LEA in most of the studies. Statistically important of associated complications like neuropathy, smoking, and osteomyelitis could not be determined. The study gives details for primary care and expert practitioners to seek for diabetic patients at increased risk for lower extremity amputation. Among the risk factors for LEA mentioned in our study, smoking, alcohol, and PVD are preventable factors.

\section{References}

1. Shin JY, Roh S-G, Sharaf B, Lee N-H. Risk of major limb amputation in diabetic foot ulcer and accompanying disease: a meta-analysis. J Plast Reconstr Aesthetic Surg 2017;70(12):1681-1688. DOI: 10.1016/ j.bjps.2017.07.015.

2. Aziz Z, Lin WK, Nather A, Huak CY. Predictive factors for lower extremity amputations in diabetic foot infections. Diabet Foot Ankle 2011. 2. DOI: $10.3402 / d f a . v 2 i 0.7463$.

3. Uçkay I, Gariani K, Pataky Z, Lipsky BA. Diabetic foot infections: stateof-the-art. Diabetes Obes Metab 2014;16(4):305-316. DOI: 10.1111/ dom.12190.

4. Shojaiefard A, Khorgami Z, Larijani B. Independent risk factors for amputation in diabetic foot. Int J Diabetes Dev Ctries 2008;28(2): 32-37. DOI: 10.4103/0973-3930.43096.

5. Diabetic Foot Infections - American Family Physician [Internet]. [cited 2020 Jan 11]. Available from: https://www.aafp.org/afp/2013/0801/ p177.html.

6. Prompers L, Huijberts M, Apelqvist J, Jude E, Piaggesi A, Bakker K, et al. High prevalence of ischaemia, infection and serious comorbidity in patients with diabetic foot disease in Europe. Baseline results from the Eurodiale study. Diabetologia 2007;50(1):18-25. DOI: 10.1007/ s00125-006-0491-1.
7. Lipsky BA, Berendt AR, Deery HG, Embil JM, Joseph WS, Karchmer AW, et al. Diagnosis and treatment of diabetic foot infections. Clin Infect Dis 2004;39(7):885-910. DOI: 10.1086/424846.

8. Lavery LA, Peters EJG, Armstrong DG. What are the most effective interventions in preventing diabetic foot ulcers? Int Wound J 2008;5(3):425-433. DOI: 10.1111/j.1742-481X.2007.00378.x.

9. Armstrong DG, Boulton AJM, Bus SA. Diabetic foot ulcers and their recurrence. Ingelfinger JR, editor. N Engl J Med 2017;376(24): 2367-2375.

10. Wukich DK, Hobizal KB, Sambenedetto TL, Kirby K, Rosario BL. Outcomes of osteomyelitis in patients hospitalized with diabetic foot infections. Foot Ankle Int 2016;37(12):1285-1291. DOI: 10.1177/1071100716664364.

11. Cervantes-García E, Salazar-Schettino PM. Clinical and surgical characteristics of infected diabetic foot ulcers in a tertiary hospital of Mexico. Diabet Foot Ankle 2017;8(1):1367210. DOI: 10.1080/2000625X.2017.1367210.

12. Mehmood K, Akhtar ST, Talib A, Talib A, Abbasi B, Siraj-ul-Salekeen, et al. Clinical profile and management outcome of diabetic foot ulcers in a tertiary care hospital. J Coll Physicians Surg Pak 2008;18(7): 408-412.

13. Uysal S, Arda B, Taşbakan MI, Çetinkalp Ş, Şimşir IY, Öztürk AM, et al. Risk factors for amputation in patients with diabetic foot infection: a prospective study. Int Wound J 2017;14(6):1219-1224. DOI: 10.1111/ iwj.12788.

14. Al-Maskari F, El-Sadig M. Prevalence of risk factors for diabetic foot complications. BMC Fam Pract 2007;8:59. DOI: 10.1186/1471-2296-859.

15. Casellini $\mathrm{CM}$, Vinik Al. Clinical manifestations and current treatment options for diabetic neuropathies. Endocr Pract 2007;13(5):550-566. DOI: 10.4158/EP.13.5.550.

16. Van Damme H, Limet R. Amputation in diabetic patients. Clin Podiatr Med Surg 2007;24(3):569-582. DOI: 10.1016/j.cpm.2007.03.007.

17. Alwakeel JS, Isnani AC, Alsuwaida A, AlHarbi A, Shaikh SA, AlMohaya $S$, et al. Factors affecting the progression of diabetic nephropathy and its complications: a single-center experience in Saudi Arabia. Ann Saudi Med 2011;31(3):236-242. DOI: 10.4103/0256-4947.81528.

18. Deshpande AD, Harris-Hayes M, Schootman M. Epidemiology of diabetes and diabetes-related complications. Phys Ther 2008;88(11):1254-1264. DOI: 10.2522/ptj.20080020.

19. Formosa C, Gatt A, Chockalingam N. Screening for peripheral vascular disease in patients with type II diabetes in Malta in a primary care setting. Qual Prim Care 2012;20(6):409-414.

20. Dalla Paola L, Faglia E. Treatment of diabetic foot ulcer: an overview strategies for clinical approach. Curr Diabetes Rev 2006;2(4):431-447. DOI: 10.2174/1573399810602040431. 